\title{
The Pivotal Role of Prevention Science in This Syndemic
}

\author{
Diana Fishbein ${ }^{1,2,3}$ [D \\ Accepted: 14 October 2020 / Published online: 19 November 2020 \\ (C) Society for Prevention Research 2020
}

\begin{abstract}
Never before has the value of prevention science become so apparent to the populace, particularly in simultaneous fashion across all nations. A general understanding of what prevention represents in true form has lagged well behind the science and, in fact, few outside of the field recognize that there is actually a significant body of research that undergirds preventative practices, programs and policies. The current pandemic and the uneven impacts on underserved and marginalized populations has highlighted the need for proactive approaches to prevent underlying conditions that increase risk for infection, worsen the wide ranging harms from the virus, and significantly exacerbate disparities that characterize many nations. To ensure uptake of the science by end-users (e.g., community stakeholders, practitioners, policymakers), who operate the levers that determine whether resources and services are distributed equitably across societies' sectors, prevention scientists have a unique and powerful role to play. This commentary on the special issue, focused on the "culture of prevention," considers the broader issues covered in the set of original articles in light of the ongoing COVID-19 pandemic. Toward that end, I also outline two interrelated "calls to action" for prevention scientists. The first call is to concertedly apply a race equity lens to all aspects of our research, a need that is particularly critical given that our field is inherently actionable and, as such, evidence amassed has potential to equalize the playing field for disadvantaged and marginalized groups. The second acknowledges the need for prevention scientists to learn how to effectively communicate scientific knowledge to the public and policymakers to compellingly advocate for reforms guided by the science. A powerful, research-backed collective advocacy can effectively sway action of governing bodies in addressing disparities and inequities for constituents who have no voice.
\end{abstract}

At no time in history has the value of prevention science become so evident to the world than in the midst of a syndemic (e.g., multiple crises) occurring simultaneously across all nations. The COVID-19 crisis has drawn attention to the dire need for a proactive, not reactive, approach to prevent the spread of the virus and its devastating health and economic consequences. The crisis has also highlighted ways in which Black and Brown people in the USA and various other groups in other nations have been under-served, under-

Diana Fishbein

dfishbein@unc.edu

1 National Prevention Science Coalition to Improve Lives (NPSC) http://www.npscoalition.org/

2 Translational Neuro-Prevention Research, FPG Child Development Institute, University of North Carolina, 105 Smith Level Road, Room 307, Chapel Hill, NC 27599, USA

3 Program on Translational Research on Adversity and Neurodevelopment (P-TRAN), Edna Bennett Pierce Prevention Research Center and Department of Human Development and Family Studies, The Pennsylvania State University, State College, PA, USA resourced, and frankly denigrated (Gould and Wilson 2020; Millett et al. 2020; Webb Hooper et al. 2020). As a result, minority populations are at much higher risk for infection, hospitalization, and death than predominant groups (Kim and Bostwick 2020; Wadhera et al. 2020). And the racial divide has rapidly become even more glaring, with an exacerbation of already extraordinary levels of poverty for otherwise affluent countries and soaring rates of mental health problems, substance abuse and dependence, domestic violence, child maltreatment, suicide, and educational lapses especially impacting marginalized groups (Fegert et al. 2020). In the USA, these disparities have existed for 400 years, but the pandemic has exposed the deep-seated nature of the problem for those who did not previously fully appreciate its magnitude.

Further amplifying the uneven impacts of the COVID-19 crisis, police shootings of several unarmed Black people plunged racial injustice onto the US national stage, bringing millions of people across all demographic groups to the streets and to their knees in fervent protest. Similar protests for comparable reasons, and even on behalf of the USA, are taking place in a number of different countries. Clearly, this is the time to act, the time to heal, and the time to transform both 
mindsets and systems. The convergence of these events presents the nation with an unprecedented opportunity to make deep inroads into the problems of inequalities, health disparities, poverty, systemic racism, and other inequities across sectors of our society. In essence, a "culture of prevention" is clearly called for-a movement that this special issue defines and justifies.

Prevention scientists have an especially germane role to play in mitigating the impact of the syndemic on marginalized populations. The overall objectives of prevention science are to (a) identify malleable risk and protective factors; (b) assess the effectiveness of programs, interventions, and policies that target those factors; and (c) develop an optimal means for dissemination and diffusion of that knowledge. Prevention strategies avert a problem before it emerges or worsens, avoiding adverse outcomes and their costs and enhancing conditions conducive to the health and well-being of individuals and strong families and communities. By intervening prior to onset or escalation of any given problem - whether it be at the individual level or within systems - a preventative approach can bolster underlying protective influences, leading to a cascade of positive outcomes (National Research Council and Institute of Medicine 2009).

In keeping with these objectives, prevention science has exerted significant social impacts on individuals, families, and communities over the past 30 years (Roumeliotis 2015; Sloboda and David 2020; Spoth et al. 2011). We have learned how to prevent/reduce academic failure, tobacco use, teen pregnancy, addiction, violence, mental health problems, and many other negative outcomes (Ramey 2018; Wandersman and Florin 2003; Yoshikawa et al. 2012). And we have learned how to promote healthy development, well-being, supportive parenting, and community cohesion (Stormshak et al. 2019; Stormshak et al. 2020). However, despite these remarkable successes, prevention strategies are chronically underutilized and underfunded, and as a result, there are few governance policies to institutionalize these programs, which the research suggests is warranted. In large part, this reality is due to the global lack of awareness on the part of the public and policymakers of the wealth of rigorous research findings from prevention science. Scientists can speak to these issues with authority to exert an influence and impact the social determinants of health by engaging in the active translation of our work.

The role for science in addressing these structural impediments to health and well-being of all people has never been clearer. This syndemic is presenting prevention scientists with a rare and unmistakable opening to address the legacy of inequities and racist public policies and practices on an international scale. Two pressing calls to action are presented herein. First is the need for programs of research to apply a racial equity lens. Doing so means that, at the core of our work, we actively seek to illuminate disparate outcomes and paying attention to race and ethnicity while analyzing the phenomena under study, identifying solutions, and developing novel and more inclusive approaches to defining success. In responding to this call, it is fundamentally critical to elevate voices of silenced constituents and communities and, in essence, nurture a co-equal partnership for the co-creation of solutions. Second is the call for researchers to learn how to effectively communicate scientific knowledge to the public and to advocate for reforms based on the science in the policy arena.

This commentary leverages some of the key take aways from the papers in this special issue and highlights the role prevention scientists can and should play in broadening the reach and practical significance of our work given its enormous potential to exert positive impacts on the phenomenon we study, at scale. This objective can be achieved, in part, by embracing approaches to prevention research that, at its core, is sensitive to race, that confronts and attempts to alleviate racial injustice, and that delineates effective methods for science advocacy. The recommendations outlined here are not fully representative of the full range of research and advocacy activities that prevention scientists can adopt to pave the way for science to advance equitable practices and policies, but they offer a starting point. Overall, the message is that prevention scientists should feel secure and defensible in taking bold action to measurably advance social, health, and racial equity amid this syndemic.

\section{Adaptations to Research Agendas}

The research agendas of prevention scientists are geared toward multilevel solution-based strategies that are inherently actionable (Antle et al. 2012). As such, it is incumbent upon us to ensure that the "end-users" (e.g., practitioners, community stakeholders, policymakers, agency administrators) are aware of the field consensual knowledge and practices we have amassed, that drivers are in place to implement those practices into well-oiled delivery systems, and that policymakers are compelled to institutionalize what works in their districts. Engaging in the work needed to entrench prevention science into mindsets, practices, and policies is our highest calling. Ultimately, the extra effort it requires to accomplish these objectives will foster inclusiveness, diversity, and equity in our work and in our ecosystem. The following promptings speak to ways we can integrate this track into our career trajectories.

First, there is a need to develop well-tested protocols that (a) identify thresholds for translating evidence (e.g., sufficiency of the evidence, criteria for designating programs as evidence-based, field consensual knowledge), (b) instill effective communications skills, (c) teach strategies for interacting with different constituent groups (e.g., segments of the population, policymakers, community stakeholders), and (d) guide 
exercises to map legislative agendas to the available evidence. Learning how to effectively advocate for and support a careful, thoughtful, and evidence-based policy approach will facilitate widespread adoption and implementation of demonstrated prevention strategies and concepts.

Second, it is critical that we concertedly diversify the scientific research community which historically addresses phenomena that are outgrowths of racial and ethnic disparities. As such, ensuring that this community is representative of, and sensitive to, the diversity of populations (e.g., vulnerable, stigmatized, oppressed), focal concerns (e.g., disparities and inequalities), and contexts (e.g., poverty and other adverse conditions) is incumbent upon the field. Prevention science is currently underrepresented by Black and Brown investigators and practitioners, as reflected in the proportion that comprises the membership of the Society for Prevention Research (SPR, 2019: 62\% Caucasian, 9.58\% Asian, 5.10\% Black, 10.32\% Hispanic).

Third, research is needed to develop evidence-based strategies that build political will and support for programs and policies that increase social equity and welfare. Strategies to move the needle down this track include developing methods to raise awareness of the pervasiveness of inequities in health and promoting empathy and support for addressing them. In parallel, studies should focus on determining how to effectively increase capacity of individuals and communities to participate in intervention efforts. The work of community coalitions - composed of agencies, organizations, faith groups, and citizens - exemplifies this process of collective action designed to strengthen the social fabric (Lardier Jr. et al. 2019). And advancing and integrating the work of implementation scientists will help us to delineate best practices for imbedding large-scale efforts to reduce racial prejudice, ideologies, and stereotypes in the larger culture that frame policy preferences that underlie and perpetrate inequities.

Fourth, intervention evaluation studies can begin to model measures of implicit bias, perceptions and experiences of racism, and racist practices as outcomes. To date, very few studies examine whether effective preventive interventions reduce racism or racist practices; only interventions that are specifically designed to directly address the phenomena include these measures as outcomes (Cobbinah and Lewis 2018; Lai et al. 2014). It is possible, for example, that preventive interventions targeting other outcomes (e.g., substance use, violence, community cohesion, academic performance) may directly affect deficits in self-image due to perceptions of racism as social emotional and competency skills improve. Or intervention-related improvements may indirectly impact the implicit biases of other players in the recipients' sphere of influence. A key question is, as the playing field is increasingly equalized, do racially driven attitudes, behaviors, and practices change accordingly? And relatedly, does (or can) the needle move in system-level practices and policies in response to the benefits demonstrated by preventive strategies? To date, research has not concertedly addressed the question of residual effects of intervention on higher-order processes. Therefore, we cannot cite evidence of the impacts of preventive intervention on the many facets and manifestations of racism or racist practices relative to individual or global attitudes, actions, or systems reforms.

Fifth, the bread and butter of prevention research has historically been on the development, implementation, and scaling of interventions. The success of this agenda as described above is remarkable. However, increasing and sustaining those impacts can be achieved by broadening the scope and scale of evidence-based interventions and focusing greater attention on identifying or developing effective methods that advance the institutionalization of those that are most efficacious and generalizable. Constraints are, in part, a function of the reality that the research process is largely dictated by timelimited grants.

On the other hand, the normalization of prevention principles and practices - otherwise called a culture of prevention in this special issue - has the greatest potential to achieve population level effects, ultimately equalizing the playing field for all segments of society. Normalization can be facilitated in two ways. First, incorporating into our daily lives the practices and principles that undergird intervention impacts - the "kernels" or active ingredients - can transform the way adults interact with each other and their children (Embry 2011). These fundamental units of programs and interventions have been shown experimentally to influence specific behaviors. Integrating a wide range of these practices and principles into daily interactions has potential to more broadly and sustainably promote health and well-being, independent of any particular intervention or grant.

The second interrelated aspect of normalization involves increasing knowledge; changing attitudes and mindsets extends from a better understanding of the science of human development and the fundamental importance of the manner in which adults interact with each other and their children (Marteau 2018). Making scientific knowledge widely accessible catalyzes change in attitudes and behaviors. Recipients of this information then become change agents themselves as they diffuse this new information to their associates, organizations, and systems within which they interact, and they disseminate it throughout their spheres of influence. As suggested by several of the papers in this special issue, the end goal should be to spur a shift in cultures, priorities, and practices that, in turn, influence policies, distribution of resources, and system level relationships. For example, incorporating these principles and practices into cross-sector service delivery systems substantially expands the scale at which benefits are achieved.

Sixth, and perhaps of greatest importance, more attention to tackling the underlying sources of exposures to social 
determinants of poor health outcomes has potential to exert broader impacts on the phenomena we seek to prevent than solely focusing on the attenuation of individual and family level adverse consequences of adversity. While the latter approach is no doubt critical, increased investments in scienceinformed practices and policies to reduce systemic inequalities, poverty, marginalization, and discrimination and to promote health equity and social welfare altogether promise to exert wide-scale impacts (Cogburn 2019; Griffith et al. 2010; Williams and Cooper 2019).

Prevention scientists, by nature, have already embraced an upstream strategy (e.g., programs that prevent substance use in adolescents, provide early education, strengthen skills to resist poor developmental outcomes, and support positive mental health) to avoid downstream costs (e.g., the financial and human burden to communities associated with treating drug addiction, juvenile delinquency, involvement in the criminal justice system, and school dropout). And when implemented effectively, the application of our well-tested practices and policies can lead to substantial cost-savings (Crowley et al. 2018). Focusing our attention further up the stream, beyond the individual, family, and even the community level, we find the roots of the problems we strive to solve in cultural, economic, linguistic, attitudinal, and structural sources. Directing our energies toward these fundamental streams of influence promises to produce transformational results.

\section{Building Bridges Between Scientists, Policymakers and the Public}

Imbedding a culture of prevention into the mindsets of the public and the decision-making process of policymakers requires that scientists systematically convey the relevance and importance of prevention; doing so will reduce the burden of phenomena we aim to prevent, minimize errors, lower costs, narrow disparities, and improve outcomes for all people of all backgrounds and at all life stages. We need an empirically tested science-to-policy protocol for experts, practitioners, and advocates across fields and sectors to increase public understanding of prevention and motivate people to demand systems and a culture that supports it. And it is essential that scientists are comfortable in this "end-stage" (scienceinformed policy reform) translational role. To facilitate this end goal, a process and vehicle must be in place to allow for clear articulation in lay terms of how research can be used to create and demonstrate practical prevention strategies and their cost effectiveness.

Prevention scientists should feel emboldened to influence policy for the betterment of society. In general, scientific evidence should and does contribute to the effectiveness of policies, a reality that legitimizes ongoing feedback between the public, researchers, and lawmakers to assure acceptability, feasibility, cultural relevance, and uptake of resultant policies (Bekker et al. 2020). The field of prevention has amassed a large body of field-consensual knowledge about the prevention of virtually every common and costly health-related problem. And a considerable arsenal of evidence-based interventions - at the individual, family, school, and community levels - has been generated. Prevention systems housing a menu of effective programs have also been imbedded in some communities for systematic delivery to populations who stand to significantly benefit (Kim et al. 2015; Spoth et al. 2017). Legislative processes can support the implementation and scale-up of these evidence-based programs and policies in communities across all segments of society.

Innovations in communications science play a critical role in the translational research process (Kendall-Taylor and Levitt 2017). After two decades of communications research, it has become apparent that a primary reason for our inability to prevent preventable conditions is that the public is unaware of, underappreciates, or discounts the power of prevention and thus does not prioritize it within a policy arena (Bromme and Beelmann 2018). Prevention researchers working closely with communications specialists and community stakeholders will be able to identify heretofore unexplored channels to actuate the results of the research.

For example, one research question relevant to health disparities is, how can social-change communicators best promote conversations about race and racism in ways that help people understand and encourage them to act and support solutions that advance equity? And throughout the communications process, it is vital that all voices and perspectives are heard by change agents. Systems change only works when citizens are engaged and connected with local community leaders and policymakers.

Shifting narratives to more effectively communicate the importance and utility of the knowledge derived from prevention science can lead to positive effects on so many levels. Moreover, since marginalized populations are especially hard hit, conveying information to key stakeholders about the dramatic effects of these strategies on health and well-being of vulnerable populations provides these otherwise quiet constituents with a voice. Prevention must become part of the American zeitgeist, as firmly entrenched as pragmatism and as cognitively accessible as health promotion.

In the advocacy realm, the work should focus on what policy reforms are needed to begin to weed out inequities and racism. The best way to direct attention to these priorities and compel action to reduce inequalities is for society to focus on common ground, highlight our interconnectedness, and foster solidarity. In the wake of the COVID-19 crisis, our collective ability to rebound from the devastation due to the pandemic may ultimately hinge on protecting and equipping our most 
vulnerable racial-ethnic minority groups and any susceptible individuals within those populations. A multitude of foundations and advocacy networks are stepping up and advancing messages that unite and propel us toward a better future. It is, in part, our professional responsibility to bolster the movement in scientific grounding.

Prevention scientists are ideally well positioned to exert an influence, all committed to be an accountable actor and supportive ally in systematically eliminating racial inequity and a broad range of other inequalities. Directing our energy into compelling deep change in mindsets, systems, and policies has the greatest potential to level the playing field for all marginalized and underresourced populations. We are seeing conclusive evidence that a powerful collective advocacy bringing thoughtful and deliberate intent to policy forums can effectively sway action of all governing bodies as it relates to health crises like the current pandemic (Michaud-Létourneau et al. 2019), which is deeply encouraging.

\section{Compliance with Ethical Standards}

Conflict of Interest The author declares that she has no conflicts of interest.

\section{Ethical Approval N/A}

Informed Consent N/A

\section{References}

Antle, B. F., Christensen, D. N., van Zyl, M. A., \& Barbee, A. P. (2012). The impact of the solution based casework (SBC) practice model on federal outcomes in public child welfare. Child Abuse \& Neglect, 36, 342-353. https://doi.org/10.1016/j.chiabu.2011.10.009.

Bekker, M., Ivankovic, D., \& Biermann, O. (2020). Early lessons from COVID-19 response and shifts in authority: Public trust, policy legitimacy and political inclusion. The European Journal of Public Health, 30(5), 854-855. https://doi.org/10.1093/eurpub/ckaa181.

Bromme, R., \& Beelmann, A. (2018). Transfer entails communication: The public understanding of (social) science as a stage and a play for implementing evidence-based prevention knowledge and programs. Prevention Science, 19, 347-357. https://doi.org/10.1007/s11121016-0686-8.

Cobbinah, S. S., \& Lewis, J. (2018). Racism \& health: A public health perspective on racial discrimination. Journal of Evaluation in Clinical Practice, 24, 995-998. https://doi.org/10.1111/jep.12894.

Cogburn, C. D. (2019). Culture, race, and health: Implications for racial inequities and population health. The Milbank Quarterly, 97, 736761. https://doi.org/10.1111/1468-0009.12411.

Crowley, D. M., Dodge, K. A., Barnett, W. S., Corso, P., Duffy, S., Graham, P., Greenberg, M., Haskins, R., Hill, L., Jones, D. E., Karoly, L. A., Kuklinski, M. R., \& Plotnick, R. (2018). Standards of evidence for conducting and reporting economic evaluations in prevention science. Prevention Science, 19, 366-390. https://doi. org/10.1007/s11121-017-0858-1.

Embry, D. D. (2011). Behavioral vaccines and evidence-based kernels: Nonpharmaceutical approaches for the prevention of mental, emotional, and behavioral disorders. The Psychiatric Clinics of North America, 34, 1-34. https://doi.org/10.1016/j.psc.2010.11. 003.

Fegert, J. M., Vitiello, B., Plener, P. L., \& Clemens, V. (2020). Challenges and burden of the coronavirus 2019 (COVID-19) pandemic for child and adolescent mental health: A narrative review to highlight clinical and research needs in the acute phase and the long return to normality. Child and Adolescent Psychiatry and Mental Health, 14, 20. https://doi.org/10.1186/s13034-020-00329-3.

Gould, E., \& Wilson, V. (2020). Black workers face two of the most lethal preexisting conditions for coronavirus-Racism and economic inequality.

Griffith, D. M., Johnson, J., Ellis, K. R., \& Schulz, A. J. (2010). Cultural context and a critical approach to eliminating health disparities. Ethnicity \& Disease, 20, 71-76.

Kendall-Taylor, N., \& Levitt, P. (2017). Beyond hat in hand: Science advocacy is foundational for policy decisions. Neuron, 94, 708712. https://doi.org/10.1016/j.neuron.2017.04.039.

Kim, S. J., \& Bostwick, W. (2020). Social vulnerability and racial inequality in COVID-19 deaths in Chicago. Health Education \& Behavior, 47, 509-513. https://doi.org/10.1177/109019812092 9677.

Kim, B. K., Gloppen, K. M., Rhew, I. C., Oesterle, S., \& Hawkins, J. D. (2015). Effects of the communities that care prevention system on youth reports of protective factors. Prevention Science, 16, 652662. https://doi.org/10.1007/s11121-014-0524-9.

Lai, C. K., Marini, M., Lehr, S. A., Cerruti, C., Shin, J. E., Joy-Gaba, J. A., Ho, A. K., Teachman, B. A., Wojcik, S. P., Koleva, S. P., Frazier, R. S., Heiphetz, L., Chen, E. E., Turner, R. N., Haidt, J., Kesebir, S., Hawkins, C. B., Schaefer, H. S., Rubichi, S., Sartori, G., Dial, C. M., Sriram, N., Banaji, M. R., \& Nosek, B. A. (2014). Reducing implicit racial preferences: I. a comparative investigation of 17 interventions. Journal of Experimental Psychology. General, 143, 1765-1785. https://doi.org/10.1037/a0036260.

Lardier Jr., D. T., Bergeson, C., Bermea, A. M., Herr, K. G., Forenza, B., Garcia-Reid, P., \& Reid, R. J. (2019). Community coalitions as spaces for collective voice, action, and the sharing of resources. Journal of Community Psychology, 47, 21-33. https://doi.org/10. 1002/jcop.22096.

Marteau, T. M. (2018). Changing minds about changing behaviour. Lancet, 391, 116-117. https://doi.org/10.1016/s0140-6736(17) 33324-x.

Michaud-Létourneau, I., Gayard, M., Mathisen, R., Phan, L. T. H., Weissman, A., \& Pelletier, D. L. (2019). Enhancing governance and strengthening advocacy for policy change of large collective impact initiatives. Maternal \& Child Nutrition, 15 Suppl 2, e12728. https://doi.org/10.1111/mcn.12728.

Millett, G. A., Jones, A. T., Benkeser, D., Baral, S., Mercer, L., Beyrer, C., Honermann, B., Lankiewicz, E., Mena, L., Crowley, J. S., Sherwood, J., \& Sullivan, P. S. (2020). Assessing differential impacts of COVID-19 on black communities. Annals of Epidemiology, 47, 37-44. https://doi.org/10.1016/j.annepidem.2020.05.003.

National Research Council \& Institute of Medicine. (2009). Preventing mental, emotional, and behavioral disorders among young people: Progress and possibilities. Washington D.C.: The National Academies Press. https://doi.org/10.17226/12480.

Ramey, C. T. (2018). The abecedarian approach to social, educational, and health disparities. Clinical Child and Family Psychology Review, 21, 527-544. https://doi.org/10.1007/s10567-018-0260-y.

Roumeliotis, F. (2015). Politics of prevention: The emergence of prevention science. The International Journal on Drug Policy, 26, 746754. https://doi.org/10.1016/j.drugpo.2015.03.011.

Sloboda, Z., \& David, S. B. (2020). Commentary on the Culture of Prevention. Prev Sci, 1-7. https://doi.org/10.1007/s11121-02001158-8. 
Spoth, R. L., Schainker, L. L., \& Hiller-Sturmhöefel, S. (2011). Translating family-focused prevention science into public health impact. Alcohol Research \& Health, 34, 188-203.

Spoth, R., Redmond, C., Shin, C., Greenberg, M. T., Feinberg, M. E., \& Trudeau, L. (2017). PROSPER delivery of universal preventive interventions with young adolescents: Long-term effects on emerging adult substance misuse and associated risk behaviors. Psychological Medicine, 47, 2246-2259. https://doi.org/10.1017/ s0033291717000691.

Stormshak, E., Caruthers, A., Chronister, K., DeGarmo, D., Stapleton, J., Falkenstein, C., DeVargas, E., \& Nash, W. (2019). Reducing risk behavior with family-centered prevention during the young adult years. Prevention Science, 20, 321-330. https://doi.org/10.1007/ s11121-018-0917-2.

Stormshak, E. A., McIntyre, L. L., Garbacz, S. A., \& Kosty, D. B. (2020). Family-centered prevention to enhance parenting skills during the transition to elementary school: A randomized trial. Journal of Family Psychology, 34, 122-127. https://doi.org/10.1037/ fam0000570.

Wadhera, R. K., Wadhera, P., Gaba, P., Figueroa, J. F., Joynt Maddox, K. E., Yeh, R. W., \& Shen, C. (2020). Variation in COVID-19 hospitalizations and deaths across New York City boroughs. JAMA, 323, 2192-2195. https://doi.org/10.1001/jama.2020.7197.

Wandersman, A., \& Florin, P. (2003). Community interventions and effective prevention. The American Psychologist, 58, 441-448. https://doi.org/10.1037/0003-066x.58.6-7.441.

Webb Hooper, M., Nápoles, A. M., \& Pérez-Stable, E. J. (2020). COVID-19 and racial/ethnic disparities. JAMA, 323, 2466-2467. https://doi.org/10.1001/jama.2020.8598.

Williams, D. R., \& Cooper, L. A. (2019). Reducing racial inequities in health: Using what we already know to take action. International Journal of Environmental Research and Public Health, 16. https:// doi.org/10.3390/ijerph16040606.

Yoshikawa, H., Aber, J. L., \& Beardslee, W. R. (2012). The effects of poverty on the mental, emotional, and behavioral health of children and youth: Implications for prevention. The American Psychologist, 67, 272-284. https://doi.org/10.1037/a0028015.

Publisher's Note Springer Nature remains neutral with regard to jurisdictional claims in published maps and institutional affiliations. 